\title{
Hyperbolic-elliptic model for surface wave in a pre-stressed incompressible elastic half-space
}

\author{
L.A. Khajiyeva ${ }^{a}$, D.A. Prikazchikov ${ }^{b}$, L.A. Prikazchikova ${ }^{b}$ \\ ${ }^{a}$ Al-Farabi Kazakh National University, Almaty,Kazakhstan \\ ${ }^{b}$ School of Computing and Mathematics, Keele University, Keele, Staffordshire, ST5 5BG, UK
}

\begin{abstract}
The paper aims at derivation of the asymptotic model for surface wave propagating in a pre-stressed incompressible elastic halfspace, subject to prescribed surface loading. The approach relies on the slow-time perturbation procedure, extending the previously known hyperbolic-elliptic formulations for surface waves in compressible linearly elastic solids. Within the derived model, the decay away from the surface is governed by a pseudo-static elliptic equation, whereas wave propagation is described by a hyperbolic equation on the surface. The effect of pre-stress, namely, the principal Cauchy stress $\sigma_{2}$, is investigated. Finally, an illustrative example of the Lamb problem is considered, demonstrating the efficiency of the approach.
\end{abstract}

Keywords: pre-stress, incompressible, surface wave, asymptotic, hyperbolic-elliptic

2000 MSC: 74J15, 74H10

\section{Introduction}

Propagation of surface waves in pre-stressed elastic media is well-studied, including the known contributions of Hayes \& Rivlin (1961), Chadwick \& Jarvis (1979), Dowaikh \& Ogden (1990). These were later extended in various directions. The 3D case of fibre-reinforced pre-stressed incompressible half-space was addressed in Prikazchikov \& Rogerson (2004). The effect of a thin coating layer was studied by Ogden \& Steigmann (2002); Steigmann \& Ogden (2007). The relation between the stretches in terms of the power law was suggested by Murphy \& Destrade (2009), investigating the effect of compressibility on propagation of surface waves. Among more recent contributions we cite Nam et al. (2016a,b), addressing the combined effects of layered vertical structure and multiple families of fibres, together with pre-stress.

An important approach to modelling of near-surface dynamics, developed in recent years, relies on the explicit hyperbolicelliptic model for the Rayleigh wave. It was first derived using the symbolic Lourier approach by Kaplunov \& Kossovich (2004), and then re-established in Kaplunov et al. (2006) through slow-time perturbation of the eigensolution in terms of a single harmonic function, presented in Chadwick (1976). Within the framework of the model, the decay into the interior is governed by an elliptic equation, with the propagation along the surface described by a hyperbolic equation. The model, originally formulated for an isotropic elastic half-plane, has been developed to 3D setup, allowing explicit approximate solutions for the near-resonant regimes of the 3D moving loads, see Kaplunov et al. (2013); Erbaş et al. (2017) and references therein. The approach has also been extended to bending Rayleigh-type waves propagating along the edge of a thin elastic plate in Kaplunov et al. (2016). A systematic presentation of the resulting theory for Rayleigh and Rayleigh-type waves may be found in Kaplunov \& Prikazchikov (2013, 2017). Recently, the effect of anisotropy have also been incorporated, see Nobili \& Prikazchikov (2018).

In this paper we aim at further development of the methodology, constructing the asymptotic model for surface wave, propagating in a pre-stressed incompressible elastic half-space subject to prescribed surface stresses. Following Dowaikh \& Ogden (1990), we formulate the problem in terms of the shear potential. Then, a slow-time perturbation scheme is established, revealing at leading order the eigensolution for surface wave in terms of a single plane harmonic function. Next order correction leads to a hyperbolic equation on the surface for the aforementioned harmonic function. The effect of pre-stress is then studied numerically for Varga material, revealing in particular the unbounded growth of the coefficient in the right hand side of the hyperbolic equation for surface displacement, as wave speed tends to zero, and vanishing of the same coefficient as surface wave degenerates into a shear wave. Finally, the asymptotic model is implemented for the Lamb problem, allowing an elegant explicit solution for the contribution of the surface wave pole.

\section{Governing equations}

Consider a homogeneous, incompressible, elastic body $\mathcal{B}$, possessing an unstressed configuration $\mathcal{B}_{0}$, occupying the domain $X_{2} \geq 0$. A purely homogeneous static deformation is imposed on $\mathcal{B}_{0}$, resulting in a finitely deformed equilibrium state $\mathcal{B}_{e}$, corresponding to a half-space $x_{2} \geq 0$. Then, a small timedependent motion is superimposed upon the equilibrium state $\mathcal{B}_{e}$, resulting in the current configuration $\mathcal{B}_{t}$. Thus, the position 
vector $\tilde{x}_{i}\left(X_{A}, t\right)$ in the current state $\mathcal{B}_{t}$ is given by

$$
\tilde{x}_{i}\left(X_{A}, t\right)=x_{i}\left(X_{A}\right)+u_{i}\left(x_{j}, t\right)
$$

Here the one-to-one mapping $x_{i}\left(X_{A}\right)$ defines the initial static deformation, and $u_{i}\left(x_{j}, t\right)$ stand for the components of superimposed small time-dependent motion.

The governing linearised equations of incremental motion are given by

$$
A_{\text {milk }} u_{k, l m}-p_{t, i}=\rho u_{i, t},
$$

see e.g. Pichugin \& Rogerson (2002). Here $A_{\text {milk }}$ are the components of the fourth order elasticity tensor, a comma indicates differentiation along the appropriate spatial or time variable, $\rho$ is volume mass density, and $p_{t}$ is the time-dependent incremental component of pressure arising due to the incompressibility constraint

$$
u_{i, i}=0 .
$$

We remark that the only non-zero components of the elasticity tensor are of the form $A_{i i j j}, A_{i j i j}$ or $A_{i j j i}$, see Ogden (1984) for more detail.

The linearised measure of incremental stress is given by

$$
\tau_{m i}=A_{m i l k} u_{k, l}+\bar{p} u_{m, i}-p_{t} \delta_{m i},
$$

where $\delta_{m i}$ is the Kronecker delta, and $\bar{p}$ denotes static pressure in the equilibrium configuration $\mathcal{B}_{e}$.

Throughout this paper the plane-strain assumption is adopted, i.e. the focus is on motions for which $u_{3}=0$ and the components $u_{1}, u_{2}$ are independent of $x_{3}$. The associated equations of incremental motion may be written explicitly as

$$
\begin{gathered}
A_{1111} u_{1,11}+\left(A_{1122}+A_{1221}\right) u_{2,12} \\
+A_{2121} u_{1,22}-p_{t, 1}=\rho u_{1, t t}, \\
A_{1212} u_{2,11}+\left(\begin{array}{c}
\left.A_{1122}+A_{1221}\right) u_{1,12} \\
+A_{2222} u_{2,22}-p_{t, 2}
\end{array}=\rho u_{2, t t},\right.
\end{gathered}
$$

along with the incompressibility condition

$$
u_{1,1}+u_{2,2}=0 .
$$

The boundary conditions on the surface $x_{2}=0$ are considered in the form of prescribed stresses $\mathbf{f}\left(x_{1}, t\right)=\left(f_{1}, f_{2}\right)^{T}$, i.e.

$$
\begin{aligned}
\tau_{21}=A_{2121} u_{1,2}+\left(A_{1221}+\bar{p}\right) u_{2,1} & =f_{1}, \\
\tau_{22}=A_{1122} u_{1,1}+\left(A_{2222}+\bar{p}\right) u_{2,2}-p_{t} & =f_{2} .
\end{aligned}
$$

Following the consideration in Dowaikh \& Ogden (1990), the displacement field is represented as

$$
u_{1}=\psi_{, 2}, \quad u_{2}=-\psi_{, 1},
$$

satisfying automatically the incompressibility condition, where $\psi$ is a generalisation of the scalar shear Lamé potential, known within 2D isotropic elasticity.

Rewriting (5) in terms of the potential $\psi$, and eliminating $p_{t}$, we result in

$$
\alpha \psi_{, 1111}+2 \beta \psi_{, 1122}+\gamma \psi_{, 2222}=\rho\left(\psi_{, 11 t t}+\psi_{, 22 t t}\right),
$$

where

$$
\begin{array}{r}
\alpha=A_{1212}, \quad \gamma=A_{2121}, \\
2 \beta=A_{1111}+A_{2222}-2\left(A_{1122}+A_{1221}\right) .
\end{array}
$$

We remark that the parameters $\alpha, \beta$ and $\gamma$ should satisfy the strong ellipticity conditions

$$
\alpha>0, \quad \beta+\sqrt{\alpha \gamma}>0 .
$$

Also, note a useful relation

$$
\bar{p}=A_{2121}-A_{1221}-\sigma_{2},
$$

following from a more general result in Ogden (1984), where $\sigma_{2}$ is the normal Cauchy stress component. Using the latter along with (8), the boundary conditions (7) are expressed as

$$
\begin{aligned}
\gamma \psi_{, 22}-\left(\gamma-\sigma_{2}\right) \psi_{, 11} & =f_{1}, \\
\left(2 \beta+\gamma-\sigma_{2}\right) \psi_{, 112}+\gamma \psi_{, 222}-\rho \psi_{, 2 t t} & =-f_{2,1} .
\end{aligned}
$$

\section{Perturbation scheme}

Below we focus on waves with phase velocity $c$ deviating slightly from surface wave speed $c_{R}$, motivating a small parameter

$$
\varepsilon=\left|\frac{c}{c_{R}}-1\right| \ll 1
$$

Our ultimate goal is extraction of the contribution of surface wave to the overall dynamic response. Clearly, this is meaningful provided that surface wave dominates over the bulk waves, which is typical, say, in the near-resonant regimes of moving loads or in the far-field of the load, for more detail see Kaplunov \& Prikazchikov (2017).

First, we introduce the scaling

$$
\xi=x_{1}-c_{R} t, \quad \tau=\varepsilon t,
$$

where $\tau$ is slow time. The boundary value problem (9), (12) is then rewritten as

$$
\begin{aligned}
& \gamma \psi_{, 2222}+\left(2 \beta-\rho c_{R}^{2}\right) \psi_{, \xi \xi 22}+\left(\alpha-\rho c_{R}^{2}\right) \psi_{\xi \xi \xi \xi} \\
& \quad+2 \varepsilon \rho c_{R}\left(\psi_{\xi \xi \xi \tau}+\psi_{22 \xi \tau}\right)-\varepsilon^{2} \rho\left(\psi_{\xi \xi \tau \tau}+\psi_{22 \tau \tau}\right)=0,
\end{aligned}
$$

subject to $\left(x_{2}=0\right)$

$$
\begin{gathered}
\gamma \psi_{, 22}-\left(\gamma-\sigma_{2}\right) \psi_{, \xi \xi}=f_{1}, \\
\left(2 \beta+\gamma-\sigma_{2}-\rho c_{R}^{2}\right) \psi_{, \xi \xi 2}+\gamma \psi_{, 222} \\
+2 \rho c_{R} \varepsilon \psi_{, 2 \xi \tau}-\rho \varepsilon^{2} \psi_{, 2 \tau \tau}=-f_{2,1} .
\end{gathered}
$$

Now, the potential $\psi$ is expanded as asymptotic series

$$
\psi=\frac{1}{\varepsilon}\left(\psi^{(0)}\left(\xi, x_{2}, \tau\right)+\varepsilon \psi^{(1)}\left(\xi, x_{2}, \tau\right)+\ldots\right) .
$$

Let us obtain the two-term asymptotic solution of the equation of motion (15). At leading order we have

$$
\gamma \psi_{, 2222}^{(0)}+\left(2 \beta-\rho c_{R}^{2}\right) \psi_{, \xi \xi 22}^{(0)}+\left(\alpha-\rho c_{R}^{2}\right) \psi_{\xi \xi \xi \xi}^{(0)}=0 .
$$


It is clear that provided the surface wave exists, the last PDE is elliptic, hence, it may be rearranged in operator form as

$$
\Delta_{1} \Delta_{2} \psi^{(0)}=0
$$

where

$$
\Delta_{j}=\partial_{22}+\kappa_{j}^{2} \partial_{\xi \xi}, \quad j=1,2
$$

with

$$
\gamma\left(\kappa_{1}^{2}+\kappa_{2}^{2}\right)=2 \beta-\rho c_{R}^{2}, \quad \gamma \kappa_{1}^{2} \kappa_{2}^{2}=\alpha-\rho c_{R}^{2} .
$$

Below we assume that $\kappa_{j}^{2}>0$, allowing solution of (19) as

$$
\psi^{(0)}\left(\xi, x_{2}, \tau\right)=\sum_{j=1}^{2} \psi_{j}^{(0)},
$$

where $\psi_{j}^{(0)}=\psi_{j}^{(0)}\left(\xi, \kappa_{j} x_{2}, \tau\right)(j=1,2)$ are arbitrary functions, harmonic in the first two arguments, i.e. $\Delta_{j} \psi_{j}^{(0)}=0$. In what follows we employ the Cauchy-Riemann identities for a harmonic function $u(x, k y)$, taking the form

$$
\frac{\partial u}{\partial x}=\frac{1}{k} \frac{\partial u^{*}}{\partial y}, \quad \frac{\partial u}{\partial y}=-k \frac{\partial u^{*}}{\partial x},
$$

with the asterisk denoting a harmonic conjugate.

At next order, (15) implies

$$
\begin{aligned}
\Delta_{1} \Delta_{2} \psi^{(1)} & =-2 \rho c_{R}\left(\psi_{\xi \xi \xi \tau}^{(0)}+\psi_{22 \xi \tau}^{(0)}\right) \\
& =2 \rho c_{R}\left[\left(\kappa_{1}^{2}-1\right) \psi_{1, \xi \xi \xi \tau}^{(0)}+\left(\kappa_{2}^{2}-1\right) \psi_{2, \xi \xi \xi \tau}^{(0)}\right] .
\end{aligned}
$$

Following the procedure similar to that presented in detail in Nobili \& Prikazchikov (2018), the solution for the correction $\psi^{(1)}$ may be found in the form

$$
\psi^{(1)}\left(\xi, x_{2}, \tau\right)=\sum_{j=1}^{2}\left(\psi_{j}^{(1,0)}+x_{2} e_{j} \psi_{j, \tau}^{(0)^{*}}\right)
$$

where $\psi_{j}^{(1,0)}=\psi_{j}^{(1,0)}\left(\xi, \kappa_{j} x_{2}, \tau\right)$ are once again arbitrary harmonic functions, and

$$
e_{j}=\frac{\rho c_{R}\left(\kappa_{j}^{2}-1\right)}{\gamma \kappa_{j}\left(\kappa_{m}^{2}-\kappa_{j}^{2}\right)}, \quad j, m \in\{1,2\}, j \neq m .
$$

Now, once the leading order solution (22) and the correction (25) are obtained, we proceed with analysis of boundary conditions. Substituting (17) into (16), using (22) and (25), performing standard manipulations with plane harmonic functions, and integrating the second equation with respect to $\xi$, at leading order we arrive at

$$
\mathbf{A} \Psi_{, \xi \xi}^{(0)}=\mathbf{0} .
$$

Here $\boldsymbol{\Psi}^{(0)}=\left(\psi_{1}^{(0)}, \psi_{2}^{(0)}\right)^{T}$, and the components of the matrix $\mathbf{A}$ are given by $(j=1,2)$

$$
\begin{aligned}
& a_{1 j}=\gamma\left(\kappa_{j}^{2}+1\right)-\sigma_{2}, \\
& a_{2 j}=\kappa_{j}\left(2 \beta-\rho c_{R}^{2}-\sigma_{2}+\gamma\left(1-\kappa_{j}^{2}\right)\right) .
\end{aligned}
$$

Then, the solvability of (27) dictates vanishing of the associated determinant $\operatorname{det} \mathbf{A}=0$. Separating the factor $\left(\kappa_{2}-\kappa_{1}\right)$ and making use of (21), this condition may be re-cast as

$$
\gamma\left(\alpha-\rho c_{R}^{2}\right)+\left(2 \beta+2 \gamma-2 \sigma_{2}-\rho c_{R}^{2}\right) \sqrt{\gamma\left(\alpha-\rho c_{R}^{2}\right)}=\left(\gamma-\sigma_{2}\right)^{2},
$$

which coincides with the surface wave equation, see Eq.(5.17) in Dowaikh \& Ogden (1990). In addition, conditions (27) imply a relationship between the harmonic functions $\psi_{1}^{(0)}$ and $\psi_{2}^{(0)}$ as

$$
\psi_{2}^{(0)}(\xi, 0, \tau)=\theta \psi_{1}^{(0)}(\xi, 0, \tau),
$$

where

$$
\theta=-\frac{a_{11}}{a_{12}}=-\frac{\gamma\left(\kappa_{1}^{2}+1\right)-\sigma_{2}}{\gamma\left(\kappa_{2}^{2}+1\right)-\sigma_{2}} .
$$

Note that this relation on the boundary implies a dependence over the interior, following from the corollary of the maximum principle for harmonic functions, see e.g. Sobolev (1964). Therefore,

$$
\psi_{2}^{(0)}\left(\xi, \kappa_{2} x_{2}, \tau\right)=\theta \psi_{1}^{(0)}\left(\xi, \kappa_{2} x_{2}, \tau\right) .
$$

At next order, the boundary conditions at $x_{2}=0$ yield

$$
\begin{gathered}
a_{11} \psi_{1, \xi \xi}^{(1,0)}+a_{12} \psi_{2, \xi \xi}^{(1,0)}=-f_{1}+2 \gamma\left(e_{1} \kappa_{1} \psi_{1, \xi \tau}^{(0)}+e_{2} \kappa_{2} \psi_{2, \xi \tau}^{(0)}\right) \\
a_{21} \psi_{1, \xi \xi}^{(1,0) *}+a_{22} \psi_{2, \xi \xi}^{(1,0) *}=-f_{2}-g_{1} \psi_{1, \xi \tau}^{(0) *}-g_{2} \psi_{2, \xi \tau}^{(0) *}
\end{gathered}
$$

where

$$
g_{j}=e_{j}\left(2 \beta-\rho c_{R}^{2}-\sigma_{2}+\gamma\left(1-3 \kappa_{j}^{2}\right)\right)-2 \kappa_{j} \rho c_{R} .
$$

Using (30), conditions (33) may be rearranged as

$$
\mathbf{A} \boldsymbol{\Psi}_{, \xi \xi}^{(1,0)}=\mathbf{B} \psi_{1, \xi \tau}^{(0)}-\mathbf{F},
$$

where the components of the matrix A were defined in (28), and

$$
\boldsymbol{\Psi}^{(0)}=\left(\begin{array}{c}
\psi_{1}^{(1,0)} \\
\psi_{2}^{(1,0)}
\end{array}\right), \quad \mathbf{B}=\left(\begin{array}{l}
b_{1} \\
b_{2}
\end{array}\right)=\left(\begin{array}{c}
2 \gamma\left(e_{1} \kappa_{1}+\theta e_{2} \kappa_{2}\right) \\
g_{1}+\theta g_{2}
\end{array}\right),
$$

with the forcing term $\mathbf{F}=\left(f_{1}, f_{2}^{*}\right)$. Note that since the vertical load component $f_{2}$ does not depend on $x_{2}$, the asterisk here is interpreted as a Hilbert transform. Since $\mathbf{A}$ is singular, from the solvability condition of (35) we deduce

$$
\psi_{1, \xi \tau}^{(0)}=\frac{1}{a_{21} b_{1}-a_{11} b_{2}}\left(a_{21} f_{1}-a_{11} f_{2}^{*}\right) .
$$

Employing the relation

$$
\partial_{\xi \tau}=\frac{c_{R}}{2 \epsilon}\left(\partial_{11}-\frac{1}{c_{R}^{2}} \partial_{t t}\right)+O(\epsilon),
$$

(37) may be re-cast in the original variables as a hyperbolic equation at the surface $x_{2}=0$

$$
\psi_{1,11}-\frac{1}{c_{R}^{2}} \psi_{1, t t}=\frac{2\left(a_{21} f_{1}-a_{11} f_{2}^{*}\right)}{c_{R}\left(a_{21} b_{1}-a_{11} b_{2}\right)}
$$

where $\psi_{1}=\epsilon^{-1} \psi_{1}^{(0)}$. Now, assuming $\psi \approx \epsilon^{-1} \psi^{(0)}$, we result in a hyperbolic-elliptic formulation for the auxiliary plane harmonic function $\psi_{1}\left(x_{1}-c_{R} t, \kappa_{1} x_{2}\right)$. 


\section{Asymptotic model for surface wave}

Thus, the explicit model for surface wave is expressed in terms of the function $\psi_{1}$ which should satisfy the pseudo-static elliptic equation

$$
\psi_{1,22}+\kappa_{1}^{2} \psi_{1,11}=0
$$

over the interior, along with the hyperbolic equation (39) on the boundary $x_{2}=0$.

Note that in view of (8), (22), and (32), the displacements may be expressed through $\psi_{1}$ as

$$
u_{1}\left(x_{1}, x_{2}, t\right)=\psi_{1,2}\left(x_{1}, \kappa_{1} x_{2}, t\right)+\theta \psi_{1,2}\left(x_{1}, \kappa_{2} x_{2}, t\right),
$$

and

$$
u_{2}\left(x_{1}, x_{2}, t\right)=-\psi_{1,1}\left(x_{1}, \kappa_{1} x_{2}, t\right)-\theta \psi_{1,1}\left(x_{1}, \kappa_{2} x_{2}, t\right) .
$$

Hence, (39) implies the following hyperbolic equations for surface displacements (at $x_{2}=0$ )

$$
u_{1,11}-\frac{1}{c_{R}^{2}} u_{1, t t}=-\frac{2\left(\kappa_{1}+\theta \kappa_{2}\right)\left(a_{21} f_{1,1}^{*}+a_{11} f_{2,1}\right)}{c_{R}\left(a_{21} b_{1}-a_{11} b_{2}\right)},
$$

and

$$
u_{2,11}-\frac{1}{c_{R}^{2}} u_{2, t t}=-\frac{2(1+\theta)\left(a_{21} f_{1,1}-a_{11} f_{2,1}^{*}\right)}{c_{R}\left(a_{21} b_{1}-a_{11} b_{2}\right)},
$$

In fact, hyperbolic equations for surface displacements were presented earlier in a simpler framework of linear isotropic elasticity (e.g. Eq. (98) in Kaplunov \& Prikazchikov (2017)). Indeed, for the case of vertical loading the hyperbolic equation for surface tangential displacement may be written as

$$
u_{1,11}-\frac{1}{c_{R}^{2}} u_{1, t t}=-\frac{1-\beta_{R}^{4}}{4 \mu B} f_{2,1} .
$$

In absence of primary deformation $\left(\lambda_{1}=\lambda_{2}=\lambda_{3}=1\right)$ we have $\alpha_{R}=1$, and $\beta_{R}=\kappa_{1}$. Then, due to incompressibility constraint, the constant $B$ is given by

$$
B=\beta_{R}^{4}-\beta_{R}-1+\beta_{R}^{-1} .
$$

It may be shown that for a vertical load $\left(f_{1}=0\right)$, in absence of pre-stress, equation (43) coincides with (45).

\subsection{Effect of pre-stress}

Let us now illustrate the effect of pre-stress on the coefficients in the derived hyperbolic equations. Consider, for example, the case of vertical loading, for which (43) yields

$$
u_{1,11}-\frac{1}{c_{R}^{2}} u_{1, t t}=A_{1 R} f_{2,1}
$$

with

$$
A_{1 R}=-\frac{2 a_{11}\left(\kappa_{1}+\theta \kappa_{2}\right)}{c_{R}\left(a_{21} b_{1}-a_{11} b_{2}\right)} .
$$

As known from Dowaikh \& Ogden (1990), the existence of surface wave in a pre-stressed incompressible elastic half-plane is guaranteed over the following range of the normal Cauchy stress $\sigma_{2}$,

$$
\sigma_{2}=\gamma-\sqrt{\gamma \alpha}+Z \sqrt{2 \sqrt{\gamma \alpha}(\beta+\sqrt{\gamma \alpha})}, \quad-1 \leq Z \leq 1 .
$$

The two limits of interest are the endpoints, when $Z= \pm 1$, associated with vanishing of surface wave speed and loss of stability, and also the special case $\sigma_{2}=\gamma$, corresponding to degeneration of surface wave into a shear wave. These two limits were also analyzed in Kaplunov et al. (2004) in respect of edge vibrations, leading to either unbounded increase in edge spectral density or vanishing of resonance phenomenon, respectively.

Let us investigate numerically the dependence of the coefficient $A_{1 R}$, defined in (48), on the Cauchy stress $\sigma_{2}$. For illustrative purposes, we specify the strain-energy function to Varga material, for which

$$
W=2 \mu\left(\lambda_{1}+\lambda_{2}+\lambda_{3}-3\right)
$$

where $\mu$ is the shear modulus in the reference configuration, and $\lambda_{i}(i=1,2,3)$ are the principal stretches, with $\lambda_{1} \lambda_{2} \lambda_{3}=1$ due to incompressibility, see e.g. Hill (2001).

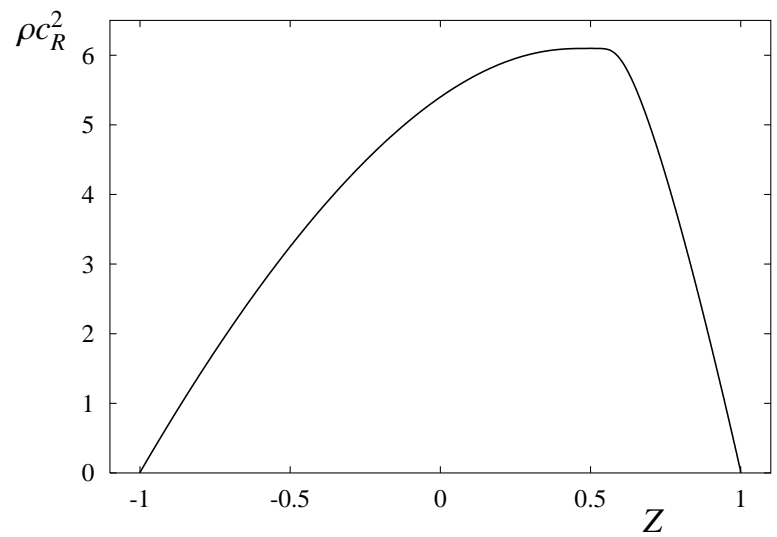

Figure 1: Effect of pre-stress on surface wave speed

Variation of the quantity $\rho c_{R}^{2}$ vs. $Z$ over the region of existence of surface wave is presented in Fig. 1, with material parameters chosen as $\mu=4.0 \mathrm{~kg} / \mathrm{cm}^{2}, \rho=1.552 \cdot 10^{-3} \mathrm{~kg} / \mathrm{cm}^{3}$, $\lambda_{1}=1.25, \lambda_{2}=0.8$, and $\lambda_{3}=1$. Note that the maximum at $\sigma_{2}=\gamma(Z \approx 0.5)$ corresponds to degeneration of surface wave.

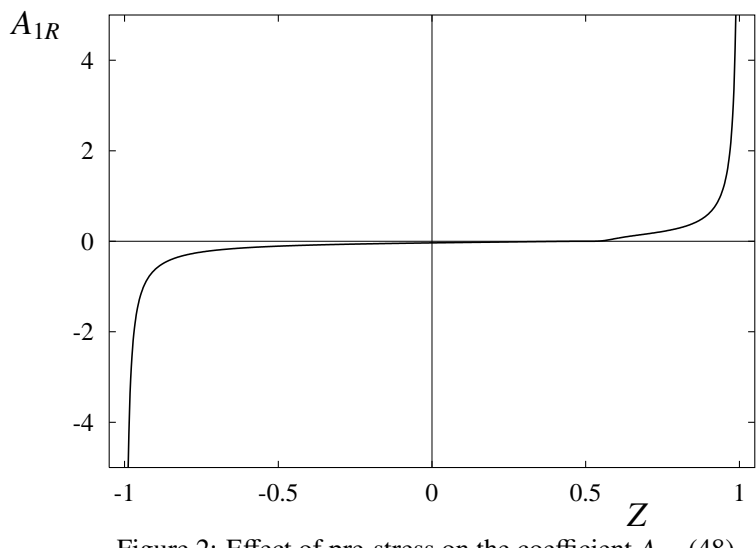

Figure 2: Effect of pre-stress on the coefficient $A_{1 R}$ (48). 
The next Fig. 2 illustrates the effect of pre-stress on the constant $A_{1 R}$ for the same material parameters as in Fig. 1. It may be observed that as $Z \rightarrow \pm 1\left(c_{R} \rightarrow 0\right)$, the coefficient $A_{1 R}$ becomes unbounded. It may be shown that in this case $A_{1 R}$ grows as $O\left(c_{R}^{-2}\right)$. Another limit $\sigma_{2} \rightarrow \gamma$ reveals that $A_{1 R}$ vanishes.

\section{Illustrative example}

Let us now demonstrate implementation of the model for a given surface load. Consider the Lamb problem, corresponding to a concentrated vertical impulse load, i.e.

$$
f_{1}=0, \quad f_{2}=P_{0} \delta\left(x_{1}\right) \delta(t) .
$$

In this case it is convenient to operate in terms of the conjugate $\psi_{1}^{*}$. Indeed, as follows from (39)

$$
\psi_{1,11}^{*}-\frac{1}{c_{R}^{2}} \psi_{1, t t}^{*}=A_{R} P_{0} \delta\left(x_{1}\right) \delta(t)
$$

with

$$
A_{R}=\frac{2 a_{11}}{c_{R}\left(a_{21} b_{1}-a_{11} b_{2}\right)} .
$$

The solution of (52) is readily obtained as

$$
\psi_{1}^{*}\left(x_{1}, 0, t\right)=\frac{c_{R} A_{R} P_{0}}{2}\left[H\left(x_{1}-c_{R} t\right)-H\left(x_{1}+c_{R} t\right)\right] .
$$

Then, the solution is restored over the interior, e.g. using the Poisson's integral formula for a half-plane, resulting in

$$
\psi_{1}^{*}\left(x_{1}, x_{2}, t\right)=\frac{c_{R} A_{R} P_{0}}{2 \pi}\left[\tan ^{-1} \frac{x_{1}-c_{R} t}{\kappa_{1} x_{2}}-\tan ^{-1} \frac{x_{1}+c_{R} t}{\kappa_{1} x_{2}}\right] .
$$

Therefore, using (41) and (42), the displacements are obtained in the form

$$
u_{j}=\frac{(-1)^{j+1}}{2 \pi} c_{R} A_{R} P_{0}\left(U_{j}^{+}-U_{j}^{-}\right),
$$

where

$$
U_{1}^{ \pm}=\frac{\kappa_{1}^{2} x_{2}}{\left(x_{1} \pm c_{R} t\right)^{2}+\kappa_{1}^{2} x_{2}^{2}}+\theta \frac{\kappa_{2}^{2} x_{2}}{\left(x_{1} \pm c_{R} t\right)^{2}+\kappa_{2}^{2} x_{2}^{2}},
$$

and

$$
U_{2}^{ \pm}=\frac{x_{1} \pm c_{R} t}{\left(x_{1} \pm c_{R} t\right)^{2}+\kappa_{1}^{2} x_{2}^{2}}+\theta \frac{x_{1} \pm c_{R} t}{\left(x_{1} \pm c_{R} t\right)^{2}+\kappa_{2}^{2} x_{2}^{2}} .
$$

\section{Conclusion}

A hyperbolic-elliptic formulation for surface wave, propagating in a pre-stressed incompressible elastic half-space, was developed. The model is expressed in terms of the auxiliary plane harmonic function $\psi_{1}$, containing a pseudo-static elliptic equation (40) over the interior, with dynamic factor arising in the hyperbolic equation (39) on the boundary. The displacement field is then expressed in terms of function $\psi_{1}$ as (41) and
(42), leading in particular to hyperbolic equations for surface displacements (43) and (44).

The effect of pre-stress was illustrated numerically for Varga strain-energy function. It was revealed that in one of the limits associated with vanishing of surface wave speed, the coefficient $A_{1 R}$ in the right hand side of the hyperbolic equation (47) tends to infinity, whereas in the second limit corresponding to degeneration of surface wave into a shear wave, $A_{1 R}$ tends to zero.

The methodology presented in this paper, could be extended further to incorporate effects of 3D and vertical inhomogeneity, see Kaplunov \& Prikazchikov (2017) for similar considerations within the linearly isotropic framework. Other exciting developments are related to seismic meta-surfaces considered by Colquitt et al. (2017) and composite models for thin plates, see Erbaş et al. (2018).

\section{Acknowledgement}

This work has been supported by the Ministry of Education and Science of the Republic of Kazakhstan, Grant IRN AP05132743. DAP acknowledges support through the Erasmus + KA107 framework. The authors are also grateful to Prof J. Kaplunov for fruitful discussions.

\section{References}

Chadwick, P., 1976. Surface and interfacial waves of arbitrary form in isotropic elastic media. Journal of Elasticity 6, 73-80.

Chadwick, P., Jarvis, D. A., 1979. Surface waves in a pre-stressed elastic body. Proceedings of the Royal Society of London A, 366, 517-536.

Colquitt, D. J., Colombi, A., Craster, R. V., Roux, P., Guenneau, S. R. L., 2017. Seismic metasurfaces: Sub-wavelength resonators and Rayleigh wave interaction. Journal of the Mechanics and Physics of Solids, 99, 379-393.

Dowaikh, M. A., Ogden, R. W., 1990. On surface waves and deformations in a pre-stressed elastic solid. IMA Journal of Applied Mathematics, 44, 261284.

Erbaş, B., Kaplunov, J., Nolde, E., Palsü, M., 2018. Composite wave models for elastic plates. Proceedings of the Royal Society of London A, 474(2214), 20180103

Erbaş, B., Kaplunov, J., Prikazchikov, D. A., Şahin, O., 2017. The near-resonant regimes of a moving load in a three-dimensional problem for a coated elastic half-space. Mathematics and Mechanics of Solids, 22(1), 89-100.

Hayes, M., Rivlin, R. S., 1961. Surface waves in deformed elastic materials. Archive for Rational Mechanics and Analysis, 8(1), 358-380.

Hill, J. M., 2001. Exact integrals and solutions for finite deformations of the incompressible Varga elastic materials. London Mathematical Society Lecture Note Series, Cambridge University Press, 160-200.

Kaplunov, Y. D., Kossovich, L. Y., 2004. Asymptotic model of Rayleigh waves in the far-field zone in an elastic half-plane. Doklady Physics 49(4), 234 236.

Kaplunov, J., Prikazchikov, D. A., 2013. Explicit models for surface, interfacial and edge waves. In: Dynamic Localization Phenomena in Elasticity, Acoustics and Electromagnetism. Springer Vienna, 73-114.

Kaplunov, J., Prikazchikov, D. A., 2017. Asymptotic theory for Rayleigh and Rayleigh-type waves. Advances in Applied Mechanics, 50, 1-106.

Kaplunov, J., Prikazchikov, D. A., Erbaş, B., Şahin, O., 2013. On a 3D moving load problem for an elastic half space. Wave Motion, 50(8), 1229-1238.

Kaplunov, J., Prikazchikov, D. A., Rogerson, G. A., 2004. Edge vibration of a pre-stressed semi-infinite strip with traction free edge and mixed face boundary conditions. ZAMP, 55, 701-719.

Kaplunov, J., Prikazchikov, D. A., Rogerson, G. A., 2016. Edge bending wave on a thin elastic plate resting on a Winkler foundation. Proceedings of the Royal Society of London A, 472, 20160178. 
Kaplunov, J., Zakharov, A., Prikazchikov, D. A., 2006. Explicit models for elastic and piezoelastic surface waves. IMA Journal of Applied Mathematics, 71, 768-782.

Murphy, J. G., Destrade, M., 2009. Surface waves and surface stability for a pre-stretched, unconstrained, non-linearly elastic half-space. International Journal of Non-Linear Mechanics, 44(5), 545-551.

Nam, N. T., Merodio, J., Ogden, R. W., Vinh, P. C., 2016. The effect of initial stress on the propagation of surface waves in a layered half-space. International Journal of Solids and Structures, 88, 88-100.

Nam, N. T., Merodio, J., Vinh, P. C., 2016. The secular equation for non-principal Rayleigh waves in deformed incompressible doubly fiberreinforced nonlinearly elastic solids. International Journal of Non-Linear Mechanics, 84, 23-30.

Nobili, A., Prikazchikov, D. A., 2018. Explicit formulation for the Rayleigh wave field induced by surface stresses in an orthorhombic half-plane. European Journal of Mechanics A/Solids, 70, 86-94.

Ogden, R. W., 1984. Non-linear Elastic Deformations. Dover, New York.

Ogden, R. W., Steigmann, D. J., 2002. Plane strain dynamics of elastic solids with intrinsic boundary elasticity, with application to surface wave propagation. Journal of the Mechanics and Physics of Solids, 50(9), 1869-1896.

Pichugin, A. V., Rogerson, G. A., 2002. An asymptotic membrane-like theory for long-wave motion in a pre-stressed elastic plate. Proceedings of the Royal Society of London A, 458, 1447-1468.

Prikazchikov, D. A., Rogerson, G. A., 2004. On surface wave propagation in incompressible, transversely isotropic, pre-stressed elastic half-spaces. International Journal of Engineering Science, 42, 967-986.

Sobolev S. L., 2016. Partial Differential Equations of Mathematical Physics: International Series of Monographs in Pure and Applied Mathematics. Pergamon Press, Oxford.

Steigmann, D. J., Ogden, R. W., 2007. Surface waves supported by thinfilm/substrate interactions. IMA Journal of Applied Mathematics, 72(6), $730-747$. 